PROCEEDINGS OF THE

AMERICAN MATHEMATICAL SOCIETY

Volume 139, Number 8, August 2011, Pages 2657-2662

S 0002-9939(2011)10695-6

Article electronically published on January 11, 2011

\title{
SKEW CATEGORIES, SMASH PRODUCT CATEGORIES AND QUASI-KOSZUL CATEGORIES
}

DEKE ZHAO

(Communicated by Martin Lorenz)

\begin{abstract}
Let $\mathscr{A}$ be a small additive Krull-Schmidt locally radical finite category over a field $K$ and let $G$ be a finite group. We show that if $\mathscr{A}$ is a free $G$-category (resp. $G$-graded category), then $\mathscr{A}$ is quasi-Koszul if and only if the skew (resp. smash product) category $G * \mathscr{A}$ (resp. $\mathscr{A} \# G$ ) is.
\end{abstract}

\section{INTRODUCTION}

Koszul algebras were originally defined by Priddy in 9 and have arisen in many contexts: algebraic geometry, Lie theory, combinatorics, non-commutative geometry and topology. There exist numerous generalizing notations of Koszul algebras. For example, Berger [2] introduced the notation of $N$-Koszul algebras, and Cassidy and Shelton [3] introduced the notation of $K_{2}$ algebras. Very recently, MartínezVilla and Solberg [7] introduced the notation of (weakly, quasi-)Koszul categories to obtain a naturally associated Koszul theory for any finite dimensional algebra.

Throughout this paper we will consider small additive categories $\mathscr{A}$ over a field $K$, free $G$-categories and $G$-graded categories where $G$ is a finite group. For $G$ categories and $G$-graded categories, Cibils and Marcos 4 defined their skew categories and smash products categories, respectively, which provide a generalized category version of the Cohen-Montgomery Duality Theorem [5]. Inspired by the closeness of the (quasi-)Koszulity of algebras under the skew products and smash products [6, 10, we show that

Theorem. Let $\mathscr{A}$ be a small additive Krull-Schmidt locally radical finite category over a field $K$ and let $G$ be a finite group.

(i) If $\mathscr{A}$ is a free $G$-category, then $\mathscr{A}$ is quasi-Koszul if and only if the skew category $G * \mathscr{A}$ is.

(ii) If $\mathscr{A}$ is G-graded, then $\mathscr{A}$ is quasi-Koszul if and only if $\mathscr{A}$ is.

In Section 2 we recall the definitions of $G$-category, of skew category, and of $G$-graded category and give some basic facts. In Section 3 we recall the definitions of the syzygy of functor and of quasi-Koszul category and prove the Theorem.

Received by the editors November 22, 2009 and, in revised form, July 12, 2010

2010 Mathematics Subject Classification. Primary 18A25, 18G10; Secondary 18B99.

Key words and phrases. Skew categories, smash product categories, quasi-Koszul categories, group-graded categories. 


\section{SKeW CATEgories AND SMASH PRODUCT CATEGORIES}

2.1. Skew categories. A $G$-category is a $K$-category $\mathscr{A}$ with firstly a set action of $G$ on the set of objects $\mathscr{A}_{0}$ and secondly $K$-module maps $g: \operatorname{Hom}_{\mathscr{A}}(X, Y) \rightarrow$ $\operatorname{Hom}_{\mathscr{A}}(g X, g Y)$ for each $g \in G$ and $(X, Y) \in \mathscr{A}_{0} \times \mathscr{A}_{0}$ satisfying $g(\varphi \psi)=(g \varphi)(g \psi)$ in case the morphism $\varphi \psi$ is composed in $\mathscr{A}$. Moreover for $g, h \in G$ and morphism $\psi$ we have $(g h) \psi=g(h \psi)$ and $e \psi=\psi$ where $e$ is the neutral element of $G$. In other words there is a group homomorphism from $G$ to the group of autofunctors of $\mathscr{A}$. If the action of $G$ is free on $\mathscr{A}_{0}$, we say that $\mathscr{A}$ is a free $G$-category.

Definition 1 (4 , Definition 2.3]). Let $\mathscr{A}$ be a $G$-category. The skew category $G * \mathscr{A}$ is a $K$-category with $(G * \mathscr{A})_{0}=\mathscr{A}_{0}$ and morphisms $\operatorname{Hom}_{G * \mathscr{A}}(X, Y)=$ $\bigoplus_{g \in G} \operatorname{Hom}_{\mathscr{A}}(g X, Y)$. Composition of morphisms is provided by the composition of $\mathscr{A}$ after adjustment.

An additive $K$-category $\mathscr{A}$ is said to be Krull-Schmidt if any object in $\mathscr{A}$ is a finite direct sum of objects with a local endomorphism algebra. Recall that the radical of a category $\mathscr{A}, \operatorname{rad} \mathscr{A}$, is a sub-bifunctor of $\operatorname{Hom}_{\mathscr{A}}(-,-)$ given by

$\operatorname{rad}_{\mathscr{A}}(X, Y)=\left\{\psi \in \operatorname{Hom}_{\mathscr{A}}(X, Y) \mid \varphi \psi \in \operatorname{rad}\left(\operatorname{End}_{\mathscr{A}}(X)\right)\right.$ for all $\left.\varphi \in \operatorname{Hom}_{\mathscr{A}}(Y, X)\right\}$.

Observe that we also have (see [8, Lemmas 4.1 and 4.2])

$\operatorname{rad}_{\mathscr{A}}(X, Y)=\left\{\psi \in \operatorname{Hom}_{\mathscr{A}}(X, Y) \mid \psi \varphi \in \operatorname{rad}\left(\operatorname{End}_{\mathscr{A}}(Y)\right)\right.$ for all $\left.\varphi \in \operatorname{Hom}_{\mathscr{A}}(Y, X)\right\}$.

We say that $\psi \in \operatorname{Hom}_{\mathscr{A}}(X, Y)$ is in $\operatorname{rad}_{\mathscr{A}}^{2}(X, Y)$ if $\psi$ is a finite sum of the form $X \stackrel{\psi_{i}^{\prime}}{\longrightarrow} X_{i}^{\prime} \stackrel{\psi_{i}^{\prime \prime}}{\longrightarrow} Y$ with $\psi_{i}^{\prime} \in \operatorname{rad}_{\mathscr{A}}\left(X, X_{i}^{\prime}\right), \psi_{i}^{\prime \prime} \in \operatorname{rad}_{\mathscr{A}}\left(X_{i}^{\prime}, Y\right)$ for $i=1, \cdots, m$ and $\psi=\sum_{i=1}^{m} \psi_{i}^{\prime \prime} \psi_{i}^{\prime}$. Inductively define $\operatorname{rad}_{\mathscr{A}}^{n}:=\operatorname{rad}_{\mathscr{A}} \cdot \operatorname{rad}_{\mathscr{A}}^{n-1}$. A $K$-category $\mathscr{A}$ is called locally radical finite if $\operatorname{rad}_{\mathscr{A}}^{i}(X, Y) / \operatorname{rad}_{\mathscr{A}}^{i+1}(X, Y)$ is finite dimensional over $K$ for all $X, Y \in \mathscr{A}$ and $i \geq 0$.

Lemma. If $X, Y \in \mathscr{A}$, then $\operatorname{rad}_{G * \mathscr{A}}(X, Y) \simeq K G \otimes_{K} \operatorname{rad}_{\mathscr{A}}(X, Y)$ and $\operatorname{rad}_{G * \mathscr{A}}^{n} \simeq$ $\overbrace{K G \otimes_{K} \cdots \otimes_{K} K G}^{n} \otimes_{K} \operatorname{rad}_{\mathscr{A}}^{n}, n \geq 1$.

Proof. Clearly $\operatorname{rad}_{\mathscr{A}}(g X, Y) \simeq \operatorname{rad}_{\mathscr{A}}(X, Y)$ for all $g \in G$. Assume that $\psi=$ $\sum_{g \in G} \psi_{g}$ and $\varphi=\sum_{h \in G} \varphi_{h}$ where $\psi_{g} \in \operatorname{Hom}_{\mathscr{A}}(g X, Y)$ and $\varphi_{h} \in \operatorname{Hom}_{\mathscr{A}}(h Y, X)$. Then $\varphi \cdot \psi \in \operatorname{rad}\left(\operatorname{End}_{G * \mathscr{A}}(X)\right)$ if and only if $\varphi_{h}\left(h \psi_{g}\right) \in \operatorname{rad}\left(\operatorname{End}_{G * \mathscr{A}}(X)\right)$ for all $g, h \in G$ if and only if $\psi_{g} \in \operatorname{rad}_{\mathscr{A}}(g X, Y)$ for all $g \in G$. Thus we obtain that

$$
\begin{aligned}
\operatorname{rad}_{G * \mathscr{A}}(X, Y)=\left\{\psi \in \operatorname{Hom}_{G * \mathscr{A}}(X, Y) \mid \varphi \psi \in \operatorname{rad}\left(\operatorname{End}_{G * \mathscr{A}}(X)\right)\right. & \left.\quad \text { for all } \varphi \in \operatorname{Hom}_{G * \mathscr{A}}(X, Y)\right\} \\
= & \left\{\psi=\sum_{g \in G} \psi_{g}, \psi_{g} \in \operatorname{Hom}_{\mathscr{A}}(g X, Y) \mid \sum_{g, h \in G} \varphi_{h} \psi_{g} \in \operatorname{rad}\left(\operatorname{End}_{G * \mathscr{A}}(X)\right)\right. \\
& \text { for all } \left.\varphi_{h} \in \operatorname{Hom}_{\mathscr{A}}(h Y, X), h \in G\right\} \\
& =\left\{\psi=\sum_{g \in G} \psi_{g}, \psi_{g} \in \operatorname{Hom}_{\mathscr{A}}(g X, Y) \mid \varphi_{h}\left(h \psi_{g}\right) \in \operatorname{rad}\left(\operatorname{End}_{G * \mathscr{A}}(X)\right)\right. \\
& \left.\quad \text { for all } \varphi_{h} \in \operatorname{Hom}_{\mathscr{A}}(h Y, X), h \in G\right\} \\
& \left\{\psi=\sum_{g \in G} \psi_{g} \mid \psi_{g} \in \operatorname{rad}_{\mathscr{A}}(g X, Y)\right\} \\
& \simeq K G \otimes_{K} \operatorname{rad}_{\mathscr{A}}(g X, Y) \\
& =K G \otimes_{K} \operatorname{rad}_{\mathscr{A}}(X, Y) .
\end{aligned}
$$


By induction, we have

$$
\begin{aligned}
\operatorname{rad}_{G * \mathscr{A}}^{n}(X, Y) & =\operatorname{rad}_{G * \mathscr{A}} \cdot \operatorname{rad}_{G * \mathscr{A}}^{n-1}(X, Y) \\
& \simeq \operatorname{rad}_{G * \mathscr{A}} \cdot \overbrace{K G \otimes_{K} \cdots \otimes_{K} K G}^{n-1} \otimes_{K} \operatorname{rad}_{\mathscr{A}}^{n-1}(X, Y)) \\
& \simeq \overbrace{K G \otimes_{K} \cdots \otimes_{K} K G}^{n} \otimes_{K} \operatorname{rad}_{\mathscr{A}}^{n}(X, Y) .
\end{aligned}
$$

2.2. Smash product categories. A G-graded category $\mathscr{B}$ is a $K$-category together with a decomposition of morphisms $\operatorname{Hom}_{\mathscr{B}}(X, Y)=\bigoplus_{g \in G} \operatorname{Hom}_{\mathscr{B}}(X, Y)_{g}$ such that $\operatorname{Hom}_{\mathscr{B}}(Y, Z)_{g} \operatorname{Hom}_{\mathscr{B}}(X, Y)_{h} \subset \operatorname{Hom}_{\mathscr{B}}(X, Z)_{g h}$. In particular, $\operatorname{Hom}_{\mathscr{B}}(X, X)$ is a $G$-graded algebra for each $X \in \mathscr{B}$.

Definition 2 (4 Definition 3.1]). Let $\mathscr{B}$ be a $G$-graded category. The smash product category $\mathscr{B} \# G$ is a category with $(\mathscr{B} \# G)_{0}=\mathscr{B}_{0} \times G$ and morphisms $\operatorname{Hom}_{\mathscr{B} \# G}((X, g),(Y, h))=\operatorname{Hom}_{\mathscr{B}}(X, Y)_{h^{-1} g}$. Composition of morphisms is provided by the graded composition of $\mathscr{B}$.

Define a $G$-action on $\mathscr{B} \# G$ by $g(X, h)=(X, g h)$ and $g f=f \in \operatorname{Hom}_{\mathscr{B} \# G}((X, g h)$, $(Y, g l))$ if $f \in \operatorname{Hom}_{\mathscr{B} \# G}((X, h),(Y, l))$. Then the category $\mathscr{B} \# G$ is a free $G$-category and the categories $G *(\mathscr{B} \# G)$ and $\mathscr{B}$ are equivalent [4, Corollary 3.3]. By the Lemma, we have the following:

Corollary. If $X, Y \in \mathscr{B}$, then $K G \otimes_{K} \operatorname{rad}_{\mathscr{B} \# G}((X, g),(Y, h))=\operatorname{rad}_{\mathscr{B}}(X, Y)$ for all $g, h \in G$.

\section{Quasi-Koszul Categories}

3.1. Syzygy of functor. Let $\mathscr{A}$ be an additive Krull-Schmidt locally radical finite $K$-category. We denote by Mod $\mathscr{A}$ the category of contravariant additive functors from $\mathscr{A}$ to the category $\operatorname{Mod} K$ of $K$-vector spaces with natural transforms as morphisms.

A family of objects $\left\{\mathcal{F}\left(Y_{i}\right) \mid Y_{i} \in \mathscr{A}, i \in I\right\}$ is said to be a family of generators for $\mathcal{F} \in \operatorname{Mod} \mathscr{A}$ if for very $Y \in \mathscr{A}$,

$$
\mathcal{F}(Y)=\sum_{i \in I} \mathcal{F}(\phi)\left(\mathcal{F}\left(Y_{i}\right)\right)
$$

where all but a finite number of $\phi_{i}: Y \rightarrow Y_{i}$ are zero, and $\mathcal{F} \in \operatorname{Mod} \mathscr{A}$ is finitely generated if it has a finite set of generator.

Denote by $\bmod \mathscr{A}$ the full subcategory of $\operatorname{Mod} \mathscr{A}$ consisting of all finitely generated contravariant additive functors of $\operatorname{Mod} \mathscr{A}$. Then the category mod $\mathscr{A}$ has, by [1, Corollary 4.13], minimal projective resolution. This gives rise to one-to-one correspondence between the indecomposable objects in $\mathscr{A}$ and the simple objects in $\operatorname{Mod} \mathscr{A}$, where an indecomposable object $X$ in $\mathscr{A}$ gives rise to the simple object $S_{X}:=\operatorname{Hom}_{\mathscr{A}}(-, X) / \operatorname{rad}_{\mathscr{A}}(-, X)$ in Mod $\mathscr{A}$. Furthermore Yoneda's Lemma implies that an $\mathscr{A}$-module is projective in $\bmod \mathscr{A}$ if and only if it is isomorphic to $\operatorname{Hom}_{\mathscr{A}}(-, X)$ for some $X \in \mathscr{A}$.

Definition 3. The syzygy functor $\Omega(\mathcal{F})$ of $\mathcal{F} \in \operatorname{Mod} \mathscr{A}$ is defined by the exact sequence

$$
0 \rightarrow \Omega(\mathcal{F}) \rightarrow \operatorname{Hom}_{\mathscr{A}}(-, X) \rightarrow \mathcal{F} \rightarrow 0
$$


for some $X \in \mathscr{A}$. Inductively, we can define the $n$-th syzygy functor $\Omega^{n}(\mathcal{F})$ of $\mathcal{F}$.

Remark. By Freyd's Theorem (see e.g. [8, p. 16, Theorem 3.1]) Mod $\mathscr{A}$ is an abelian category with coproduct and a faithful set of small projectives. Thus $\Omega(\mathcal{F}): \mathscr{A} \rightarrow$ $\operatorname{Mod} K, M \mapsto \Omega(\mathcal{F})(M)$ is a contravariant functor for any $\mathcal{F} \in \bmod \mathscr{A}$.

3.2. Quasi-Koszul categories. Recall that an ideal in the category $\mathscr{A}$ is a subbifunctor of the Hom-functor. For two ideals $\mathcal{I}$ and $\mathcal{J}$ we define in a natural way inclusion, intersection and product. In particular the product of two ideals $\mathcal{I}$ and $\mathcal{J}$ is given by

$$
\begin{aligned}
& \mathcal{I} \mathcal{J}(X, Z)=\left\{\psi \in \operatorname{Hom}_{\mathscr{A}}(X, Z) \mid \psi\right. \\
&\left.\quad=\sum_{i=1}^{n} \varphi_{i} \phi_{i}, \phi_{i} \in \operatorname{Hom}_{\mathscr{A}}\left(X, Y_{i}\right), \varphi \in \operatorname{Hom}_{\mathscr{A}}\left(Y_{i}, Z\right), Y_{i} \in \mathscr{A}\right\} \text { for } X, Z \in \mathscr{A} .
\end{aligned}
$$

For an $n$-fold product of an ideal $\mathcal{I}$ with itself we write $\mathcal{I}^{n}$. Let $\mathcal{I}$ be an ideal in the category. For a contravariant functor $\mathcal{F}: \mathscr{A} \rightarrow \operatorname{Mod} K$ define $\mathcal{I F}: \mathscr{A} \rightarrow \operatorname{Mod} K$ as the subfunctor of $\mathcal{F}$ given by

$$
\mathcal{I F}(M)=\sum_{X \in \mathscr{A}} \mathcal{F}(\mathcal{I}(M, X))
$$

for all objects $M \in \mathscr{A}$. Clearly $\mathcal{I F} \subseteq \mathcal{F}$ for any ideal $\mathcal{I}$ and any contravariant functor $\mathcal{F}$ in $\operatorname{Mod} \mathscr{A}$

Definition $4([7, \S 5])$. A contravariant functor $\mathcal{F}$ in $\operatorname{Mod} \mathscr{A}$ is quasi-Koszul if it has a finitely generated projective resolution

$$
\cdots \rightarrow \operatorname{Hom}_{\mathscr{A}}\left(-, X_{i}\right) \rightarrow \cdots \rightarrow \operatorname{Hom}_{\mathscr{A}}\left(-, X_{0}\right) \rightarrow \mathcal{F} \rightarrow 0
$$

and $\operatorname{rad}_{\mathscr{A}} \Omega^{i}(\mathcal{F})=\operatorname{rad}_{\mathscr{A}}^{2}\left(-, X_{i-1}\right) \cap \Omega^{i}(\mathcal{F})$ for all $i \geq 1$.

The category $\mathscr{A}$ is quasi-Koszul if every simple functor in Mod $\mathscr{A}$ is quasi-Koszul.

3.3. Proof of Theorem. Since the categories $G *(\mathscr{A} \# G)$ and $\mathscr{A}$ are equivalent, it is sufficient to prove (i). Assume that $\mathscr{A}$ is quasi-Koszul and $\mathcal{F}$ is a simple contravariant functor in $\operatorname{Mod} \mathscr{A}$ having a finitely generated projective resolution

$$
\cdots \rightarrow \operatorname{Hom}_{\mathscr{A}}\left(-, X_{i}\right) \rightarrow \cdots \rightarrow \operatorname{Hom}_{\mathscr{A}}\left(-, X_{0}\right) \rightarrow \mathcal{F} \rightarrow 0
$$

and that $\operatorname{rad}_{\mathscr{A}} \Omega^{i}(\mathcal{F})=\operatorname{rad}_{\mathscr{A}}^{2}\left(-, X_{i-1}\right) \cap \Omega^{i}(\mathcal{F})$ for all $i \geq 1$.

Suppose that $\mathcal{F}=\operatorname{Hom}_{\mathscr{A}}(-, X) / \operatorname{rad}_{\mathscr{A}}(-, X)$ for some indecomposable object $X \in \mathscr{A}$ and define

$$
\mathcal{F}^{*}:=\operatorname{Hom}_{G * \mathscr{A}}(-, X) / \operatorname{rad}_{G * \mathscr{A}}(-, X) .
$$

Then $\mathcal{F}^{*}$ is a simple contravariant functor in $\operatorname{Mod} G * \mathscr{A}$, and every simple functor in $\operatorname{Mod} G * \mathscr{A}$ is of this form, which has a finitely generated projective resolution

$$
\cdots \rightarrow \operatorname{Hom}_{G * \mathscr{A}}\left(-, X_{i}\right) \rightarrow \cdots \rightarrow \operatorname{Hom}_{G * \mathscr{A}}\left(-, X_{0}\right) \rightarrow \mathcal{F}^{*} \rightarrow 0 .
$$

Thus it is enough to show that

(*) for all $i \geq 1, \operatorname{rad}_{\mathscr{A}} \Omega^{i}(\mathcal{F})=\operatorname{rad}_{\mathscr{A}}^{2}\left(-, X_{i-1}\right) \cap \Omega^{i}(\mathcal{F})$

if and only if $\operatorname{rad}_{G * \mathscr{A}} \Omega^{i}\left(\mathcal{F}^{*}\right)=\operatorname{rad}_{G * \mathscr{A}}^{2}\left(-, X_{i-1}\right) \cap \Omega^{i}\left(\mathcal{F}^{*}\right)$. 
For any object $M \in \mathscr{A}$ and $i \geq 1$, we have

$$
\begin{aligned}
\operatorname{rad}_{G * \mathscr{A}} \Omega^{i}\left(\mathcal{F}^{*}\right)(M) & =\sum_{Y \in \mathscr{A}} \Omega^{i}\left(\mathcal{F}^{*}\right)\left(\operatorname{rad}_{G * \mathscr{A}}(M, Y)\right) \\
& =\sum_{Y \in \mathscr{A}} \Omega^{i}\left(\mathcal{F}^{*}\right)\left(K G \otimes_{K} \operatorname{rad}_{\mathscr{A}}(M, Y)\right) \\
& =\sum_{Y \in \mathscr{A}} K G \otimes_{K} K G \otimes_{K} \Omega^{i}(\mathcal{F})\left(\operatorname{rad}_{\mathscr{A}}(M, Y)\right) \\
& =K G \otimes_{K} K G \otimes_{K} \operatorname{rad}_{\mathscr{A}} \Omega^{i}(\mathcal{F})(M)
\end{aligned}
$$

that is, for all $i \geq 1$,

$$
\operatorname{rad}_{G * \mathscr{A}} \Omega^{i}\left(\mathcal{F}^{*}\right)=K G \otimes_{K} K G \otimes_{K} \operatorname{rad}_{\mathscr{A}} \Omega^{i}(\mathcal{F}) .
$$

By the Lemma, we have

$\operatorname{rad}_{G * \mathscr{A}}^{2}\left(-, X_{i-1}\right) \cap \Omega^{i}\left(\mathcal{F}^{*}\right)=K G \otimes_{K} K G \otimes_{K} \operatorname{rad}_{\mathscr{A}}^{2}\left(-, X_{i-1}\right) \cap \Omega^{i}\left(\mathcal{F}^{*}\right) \quad$ for all $i \geq 1$.

Since $\Omega^{i}\left(\mathcal{F}^{*}\right) \subseteq \operatorname{rad}_{G * \mathscr{A}} \operatorname{Hom}_{G * \mathscr{A}}\left(-, X_{i-1}\right)$ for all $i \geq 1$,

$$
\begin{aligned}
\Omega^{i}\left(\mathcal{F}^{*}\right)(M) & \subseteq \operatorname{rad}_{G * \mathscr{A}} \operatorname{Hom}_{G * \mathscr{A}}\left(-, X_{i-1}\right) \\
& =\sum_{Y \in \mathscr{A}} \operatorname{Hom}_{G * \mathscr{A}}\left(-, X_{i-1}\right) \operatorname{rad}_{G * \mathscr{A}}(M, Y) \\
& =K G \otimes_{K} K G \otimes_{K} \operatorname{Hom}_{\mathscr{A}}\left(-, X_{i-1}\right) \operatorname{rad}_{\mathscr{A}}(M, Y) \\
& =K G \otimes_{K} K G \otimes_{K} \operatorname{rad}_{\mathscr{A}} \operatorname{Hom}_{\mathscr{A}}\left(-, X_{i-1}\right)(M) .
\end{aligned}
$$

On the other hand, note that $\Omega^{i}(\mathcal{F}) \subseteq \operatorname{rad}_{\mathscr{A}} \operatorname{Hom}_{\mathscr{A}}\left(-, X_{i-1}\right)$. This yields that $\Omega^{i}\left(\mathcal{F}^{*}\right)=K G \otimes_{K} K G \otimes_{K} \Omega^{i}(\mathcal{F})$. Hence

$$
\operatorname{rad}_{G * \mathscr{A}}^{2}\left(-, X_{i-1}\right) \cap \Omega^{i}\left(\mathcal{F}^{*}\right)=K G \otimes_{K} K G \otimes_{K} \operatorname{rad}_{\mathscr{A}}^{2}\left(-, X_{i-1}\right) \cap \Omega^{i}(\mathcal{F}) .
$$

Now $(*)$ follows by combining (I) and (II). This completes the proof.

\section{REFERENCES}

1. M. Auslander, Representation theory of Artin algebras. I, Comm. Algebra 1(1974), 177-268. MR 0349747 (50:2240)

2. R. Berger, Koszulity for nonquadratic algebras, J. Algebra 239(2001), 705-734. MR.1832913 (2002d:16034)

3. T. Cassidy and B. Shelton, Generalizing the notion of Koszul algebra, Math. Z. 260(2008), 93-114. MR 2413345(2009e:16047)

4. C. Cibils and E.N. Marcos, Skew category, Galois covering and smash product of a $k$-category, Proc. Amer. Math. Soc. 134(2006), 39-50. MR2170541 (2006e:18003)

5. M. Cohen and S. Montgomery, Group-graded rings, smash products, and group actions, Trans. Amer. Math. Soc. 282(1984), 237-258. MR728711 (85i:16002)

6. R. Martínez-Villa, Skew group algebras and their Yoneda algebras, Math. J. Okayama Univ. 43(2001), 1-16. MR1913868 (2003g:18013)

7. R. Martínez-Villa and $\varnothing$. Solberg, Graded and Koszul categories, Appl. Categor. Struct. 18(2010), 615-652.

8. B. Mitchel, Rings with several objects, Adv. Math. 8(1972), 1-161. MR0294454 (45:3524) 
9. S.B. Priddy, Koszul resolutions, Trans. Amer. Math. Soc. 152(1970), 39-60. MR0265437 $(42: 346)$

10. D.K. Zhao and Y. Han, Koszul algebras and finite Galois coverings, Sci. China Ser. A 52(2009), 2145-2153. MR2550272

Academy of Mathematics and Systems Science, Chinese Academy of Sciences, Beijing 100190, People's Republic of China

E-mail address: deke@amss.ac.cn

Current address: School of Applied Mathematics, Beijing Normal University at Zhuhai, Zhuhai, 519087, People's Republic of China 